\title{
Evaluation of stabilization of intra-articular fracture of distal humerus - finite element study
}

\author{
ARTUR KRUSZEWSKI ${ }^{1}$, SZCZEPAN PISZCZATOWSKI ${ }^{1 *}$, \\ PIOTR PIEKARCZYK ${ }^{2}$, KRZYSZTOF KWIATKOWSKI ${ }^{2}$ \\ ${ }^{1}$ Faculty of Mechanical Engineering, Bialystok University of Technology, Białystok, Poland. \\ ${ }^{2}$ Department of Traumatology and Orthopaedics, Military Institute of Medicine, Warsaw, Poland.
}

\begin{abstract}
Purpose: The treatment of comminuted fractures of distal humerus poses a challenge for orthopaedics. Previous studies assessing the global stiffness of the bone - stabilizer system - made it impossible to explicitly indicate an optimal configuration of the locking plates in the treatment of this kind of fractures. The aim of the present research was to comparatively analyze the stabilization conditions of intraarticular fractures of distal humerus with the use of various configurations of the stabilizer. Methods: The research was based on the analysis of mutual displacements of bone fragments. Such evaluation was performed with the use of numerical simulation conducted with the use of the finite element method. A realistic model of humerus was based on the CT data. Three spatial configurations of the stabilizer (parallel, posteromedial and posterolateral) were considered. The mutual displacements of bone fragments as well as the deformity of the stabilizer under various loading conditions were analyzed. Results: In most cases, the parallel setting of the plates ensures a better stabilization of the bone fragments than the perpendicular configuration. The most difficult conditions of stabilization were obtained for the lateral bone fragment. The value of the fragments' displacements significantly increases for loading directions occurring with ascending flexion angle of the joint. Conclusions: In most cases, the parallel setting of the plates ensures a better stabilization of the bone fragments than the perpendicular configuration.
\end{abstract}

Key words: distal humerus, fracture, plate stabilization, numerical simulation, biomechanics

\section{Introduction}

Distal humeral fractures represent about $30 \%$ of fractures of the humerus and they are the cause of approximately $37 \%$ of all elbow surgeries [1]. The stabilization should provide a sufficiently stable fixation in order to obtain union. It should also allow for an early rehabilitation process as movement is essential for the final treatment success due to the fact that the elbow is intolerant of immobilization [9].

The gold standard in the treatment of distal humerus fractures is the open reduction and internal fixation, in which locking plates and screws are used
[21]. In the case of intra-articular fractures, a pair of the blocking plates is used most often [24]. Nowadays, there are two most popular plating techniques. The first one deals with parallel plating using medial and lateral plates [17], while the other refers to perpendicular plating [21] with two available options: "posterolateral" with medial and postero-lateral plates as well as "posteromedial" with lateral and posteromedial plates.

Parallel plating was the consequence of earlier reports showing unsatisfactory results among patients with perpendicular plating [9], yet, the optimal plate configuration still remains controversial, similarly to other orthopedic situations [5]. Penzkofer et al. [18],

* Corresponding author: Szczepan Piszczatowski, Bialystok University of Technology, Faculty of Mechanical Engineering, ul. Wiejska 45C, 15-351 Białystok, Poland. Phone: +48 571443085, e-mail: s.piszczatowski@pb.edu.pl

Received: October 14th, 2019

Accepted for publication: February 18th, 2020 
Schwartz et al. [22] and Arnander et al. [2] described biomechanical tests where parallel and perpendicular fixing plates systems were compared using artificial bone models. They concluded that both systems provide sufficient stiffness. Penzkofer found that parallel configuration gave a better stiffness in extension only. Schwartz found that there was no statistical difference in stiffness in any direction. Longitudinal strain for perpendicular plates configuration was significantly lesser in axial compression while parallel arrangement demonstrated a significantly lower level of transverse deformity during axial torsion.

Zalavras et al. [26], Got [8] and Koonce et al. [12] made similar tests on cadaveric distal humerus models. Zalavras revealed that the parallel plate system is more stable than the perpendicular one. The latter research showed that the perpendicular configuration of plates is comparable to the parallel one. Moreover, Got found that perpendicular plating had a greater resistance to torsional loading. O'Driscol [17] examined clinical outcomes of distal humerus fracture treatment and concluded that the parallel method provided a better fracture stabilization. Theivendran came to a similar conclusion [25]. However, Shin et al. [23] as well as Clavert et al. [6] did not observe any significant differences between parallel and perpendicular plating methods in terms of clinical outcomes and complications. Satisfying clinical results with the use of the stabilizer in perpendicular configuration were presented, among others, by Puchwein et al. [19], and Schmidt-Horlohe et al. [24].

The ambiguity of biomechanical analysis regarding the stabilizer configuration for distal humerus fractures as well as the lack of distinct indications for optimal treatment of individual patients resulted in an attempt to perform a more detailed analysis of the problem. It is well known that for proper bone union it is crucial to stabilize bone blocks to avoid their mutual movement [21]. As far as comminuted fractures are concerned, the assessment of global stiffness of the stabilizer does not give an opportunity for a realistic assessment of conditions of the union when it comes to the particular bone fragments. This might explain the ambiguity of the outcomes from the previous studies.

The aim of the research in focus was to assess the conditions of intra-articular stabilization in comminuted fractures of distal humerus on the basis of the analysis of mutual displacements of particular bone fragments for various stabilizer configurations.

\section{Materials and methods}

Modeling and numerical simulation performed with the finite element method seem to be the optimal tools for achieving the aforementioned aim of the research. It was assumed that the 13-C1 type, according to AO [21], is a sufficiently representative example for the analysis. It is characterized by the bone fracture which goes through articular surfaces in the area of the trochlea as well as by the presence of bone fragments on the medial and lateral sides.

\subsection{Geometrical model}

The geometrical model of humerus (Fig. 1) was prepared on the basis of results of the X-ray Computed Tomography (CT) of a 19-year-old woman with the 13-C1 fracture of the left arm, obtained during a routine diagnostic process. The $\mathrm{CT}$ data was processed with the use of the MIMICS software (Materialise BV, Belgium) which allowed the segmentation and the preparation of solid models of the particular bone fragments (Fig. 1a). In reconstructed bone structure it is possible to differentiate the shaft and two bone fragments located in the distal part on the medial side (hereinafter referred to as medial fragment) as well as the lateral side (hereinafter referred to as lateral fragment). The virtual reposition of the medial and lateral bone blocks with regard to the shaft was conducted. This made it possible to obtain a geometrical model of the fractured bone prepared for union (Fig. 1b).

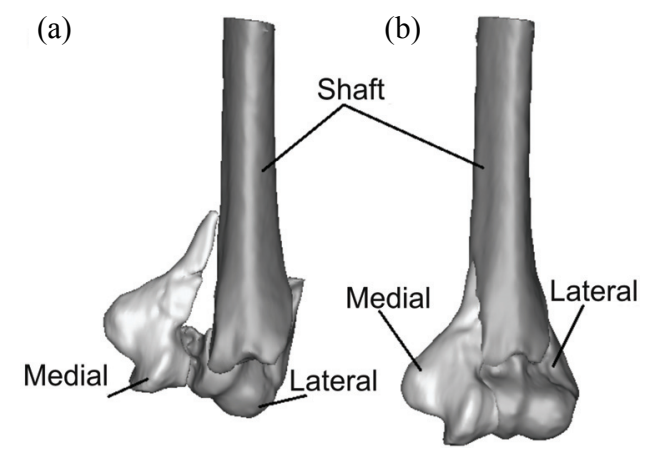

Fig. 1. Three-dimensional reconstruction of distal humerus fracture based on CT-data obtained for 19-year-old woman before (a) and after (b) virtual reposition

The model of the stabilizer was established on the basis of the VariAx elbow locking plate system (Stryker, USA). Four plates different in shape (lateral plate 
(a)
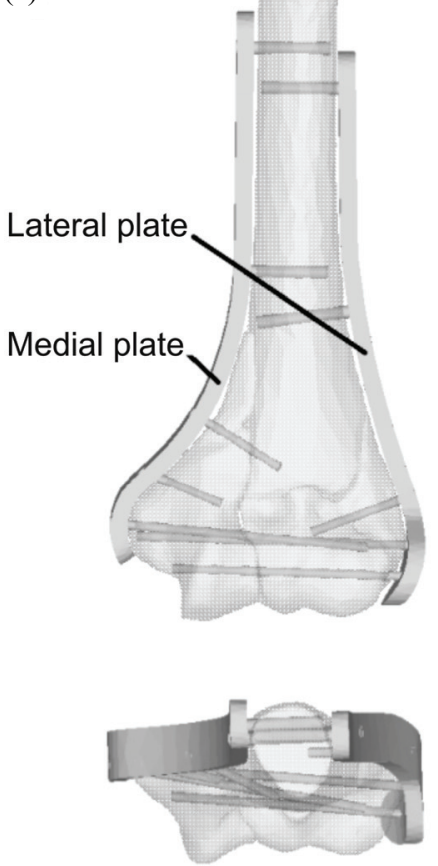

(b)
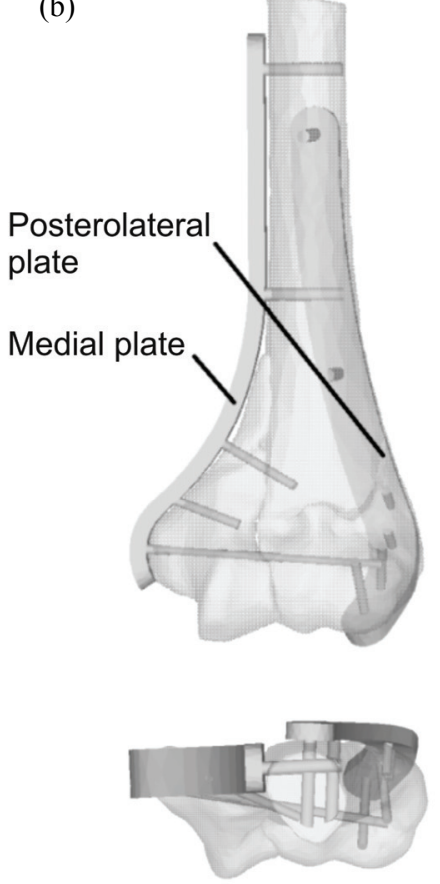

(c)

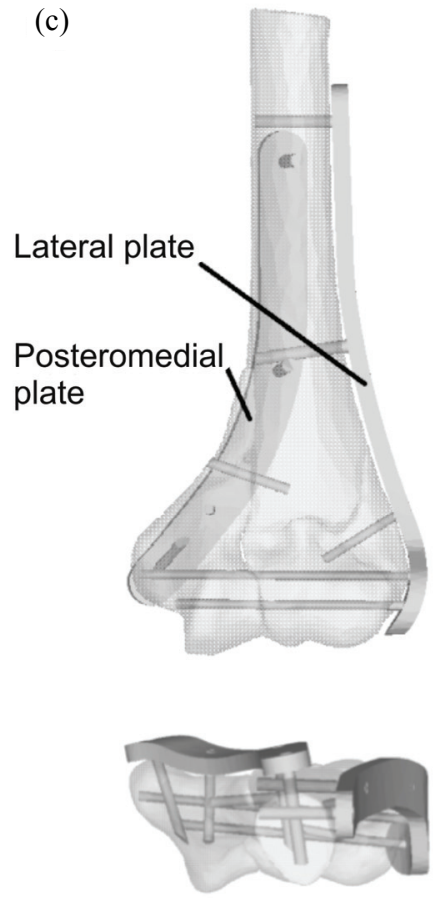

Fig. 2. The cross-section of the model of the humerus with visible regions of different material properties

- length of $109 \mathrm{~mm}$, medial plate - length of $116 \mathrm{~mm}$, osteromedial - length of $104 \mathrm{~mm}$ and posterolateral - length of $105 \mathrm{~mm}$ ), were modeled to be used on the lateral or medial sides so as to obtain three variants of the stabilizer. Geometry of the plates were simplified with the constant thickness of all plates equaled $3.5 \mathrm{~mm}$. In the "parallel" variant, particular bone fragments were stabilized with the use of medial and lateral plates located approximately in parallel to each other (Fig. 2a). In the "perpendicular" variant, the plates were oriented perpendicularly to each other. In the "posterolateral" version (Fig. 2b), a medial plate was placed in the same way as in the parallel configuration, but a posterolateral plate was used instead of a lateral plate. It was placed in the perpendicular plane to the medial plate, on the posterolateral side of the humerus. As far as "posteromedial" version is concerned (Fig. 2c), the lateral plate was located in the same way as in the parallel configuration, but the medial plate was substituted by the posteromedial one which was located on the posteromedial side of the bone. The screw system that was modeled reflected the way of insertion of the screws in the clinical practice in accordance with the $\mathrm{AO}$ rules and principles concerning the optimization of stability using screws and plates postulated by O'Driscoll [17], [21]. Their localization and spatial arrangement (Figs. $2 \mathrm{a}-\mathrm{c}$ ) were substantially different in particular stabilization variants due to a different shape of the plates and their location relative to the bone fragments. Screws con- necting the plate models with the bone model were simplified and modelled without the thread as the cylinders fully bonded to the bone in the thread region. Screws used in the shaft region were modelled as a cylinder of $2.75 \mathrm{~mm}$ in diameter, which corresponds to the core diameter of the $3.5 \mathrm{~mm}$ screw, whilst those intended for distal region of humerus were modelled as cylinders of diameter equal $2 \mathrm{~mm}$ which corresponds to the core diameter of the $2.75 \mathrm{~mm}$ screw. The discretization of solid models was made with the use of the ANSYS system (Ansys, Inc., Canonsburg, USA), using 10-nodes tetrahedral solid elements named SOLID187.

\subsection{Material modeling}

The bone tissue was significantly simplified and modeled as an isotropic, elastic material. A heterogeneous structure of the humerus was taken into account (Table 1). Its model was made of the shaft built of a quite strong cortical bone, a marrow cavity as well as an inhomogeneous distal epiphysis built mainly of a cancellous bone. On the basis of the CT data analysis, it was observed that the density of the bone tissue is highly diversified in the area of humerus epiphysis. It is consistent with what was observed by Diederichs et al. [7]. Epiphysis was divided into four regions differing in mechanical properties [13]. 
Table 1. Material properties used in the model

\begin{tabular}{|c|c|c|c|c|c|}
\hline $\begin{array}{l}\text { Material } \\
\text { type }\end{array}$ & HU & $\begin{array}{c}\rho \\
{\left[\mathrm{kg} / \mathrm{m}^{3}\right]}\end{array}$ & $\begin{array}{c}\text { Young's } \\
\text { modulus E } \\
{[\mathrm{GPa}]}\end{array}$ & $\begin{array}{c}\text { Poisson's } \\
\text { ratio }\end{array}$ & References \\
\hline $\begin{array}{l}\text { Cancellous } \\
\text { bone I }\end{array}$ & 110 & 240 & 0.75 & 0.34 & \multirow{4}{*}{ [20] } \\
\hline $\begin{array}{l}\text { Cancellous } \\
\text { bone II }\end{array}$ & 180 & 285 & 0.94 & 0.34 & \\
\hline $\begin{array}{l}\text { Cancellous } \\
\text { bone III }\end{array}$ & 200 & 298 & 1 & 0.34 & \\
\hline $\begin{array}{l}\text { Cancellous } \\
\text { bone IV }\end{array}$ & 290 & 354 & 1.23 & 0.34 & \\
\hline \begin{tabular}{|l|}
$\begin{array}{l}\text { Cortical } \\
\text { bone }\end{array}$ \\
\end{tabular} & 1400 & 1400 & 14.7 & 0.34 & [20] \\
\hline $\begin{array}{l}\text { Screws } \\
\text { \& plates } \\
(\text { Ti6Al4V) }\end{array}$ & - & - & 96 & 0.3 & [3] \\
\hline \begin{tabular}{|l|}
$\begin{array}{l}\text { Marrow } \\
\text { cavity }\end{array}$ \\
\end{tabular} & 0.001 & 0.001 & 0.001 & 0.34 & \\
\hline
\end{tabular}

For particular regions, the average apparent density $\rho$ was established on the basis of averaged radiological density for a given region, expressed in Hounsfield's units [HU], and then, the Young's modulus $E$ was established on the basis of tissue apparent density, using the formula proposed by Rho et al. [20]. Similar methods were used for the cortical bone, which was modeled for the shaft region as well as for thin layer around the epiphysis. The cortex layer in its thinnest region was $1.6 \mathrm{~mm}$ thick and has been discretized using at least two layers of tetrahedral solid elements. In the case of the plate and screws models, it was assumed that the material properties were the same as for the titanium alloy Ti6A14V [3].

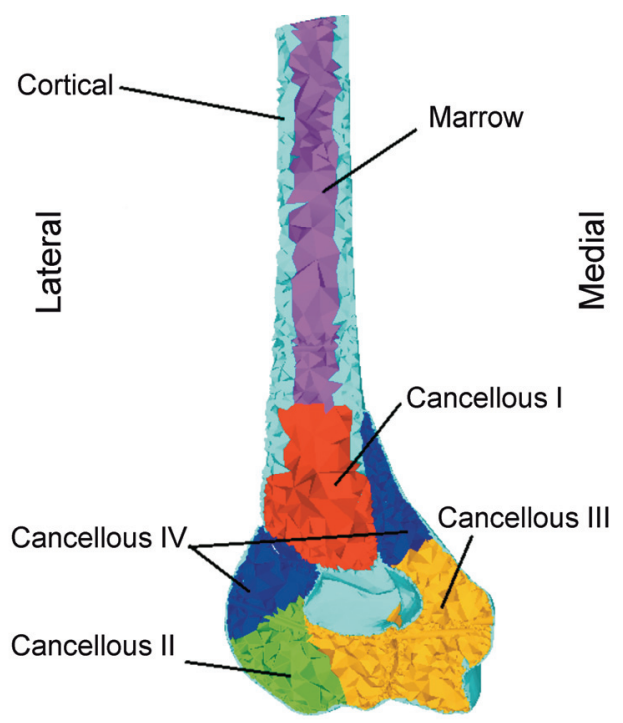

Fig. 3. The maximum mutual displacements of bone fragments in the normal (on the left) and tangential direction (on the right) as regards three methods of stabilization with respect to the flexion angle in the elbow joint:

a) region $\mathrm{A}$; b) region $\mathrm{B}$; c) region $\mathrm{C}$; $\mathrm{d}$ ) region $\mathrm{D}$
In Table 1, material properties used in the model are summarized. The distribution of the regions of occurrence of particular types of tissue is presented in Fig. 3.

\subsection{Loads and boundary conditions}

There is little information about humero-ulnar joint loads in the literature. Kincaid and An [11] summarized previous studies presenting a review of the elbow joint forces associated with activities of daily living. Analyzing these results, it can be noticed that the relation between the angle of the elbow flexion and the direction of joint reaction force (JRF) in sagittal plane in relation to the humerus axis is almost linear. In such a situation, numerical simulations were performed only for four elbow angles: variant I - fully extended position $\left(0^{\circ}\right)$, variant IV - close to maximal flexion $\left(145^{\circ}\right)$ and for two intermediate positions (variant II $-30^{\circ}$, variant III $-75^{\circ}$ ). Based on Kincaid and An analysis, there was an assumed direction of the vector of the force acting on humerus for various position of the elbow (Table 2, Fig. 4). The loads were applied on the trochlea with an appropriate balance between the forces affecting the medial and lateral fragments. The assumed value of the forces was $200 \mathrm{~N}$, which corresponds well with the activities of daily living [16] and is considered to be related to the loads acting when holding a $2 \mathrm{~kg}$ weight in one hand having the elbow joint in a straight position. In the course of the studies, an equal JRF value for different elbow joint angles was assumed, which made it possible to compare particular versions of the stabilizer regarding different load directions, without any differences resulting from different load values. Nonetheless, it should be remembered that the existing analyses point to the fact that the JRF value increases when it comes to the decreased value of the angle in the elbow [11].

Table 2. Direction of Joint Reaction Force (JRF) in sagittal planes for various elbow flexion angles [11]

\begin{tabular}{|l|c|c|c|c|}
\hline Variant of loading & I & II & III & IV \\
\hline Elbow flexion angle & $0^{\circ}$ & $30^{\circ}$ & $75^{\circ}$ & $145^{\circ}$ \\
\hline JRF angle in sagittal plane & $-20^{\circ}$ & $10^{\circ}$ & $43^{\circ}$ & $95^{\circ}$ \\
\hline
\end{tabular}

The bone model was supported in its proximal part. The stabilizer plates were connected with the shaft of the bone as well as with particular bone fragments only by the system of screws. The plates and the screws as well as the screws and the bone were fully bound to one another. Variable contact conditions were modeled between particular bone fragments using contact ele- 

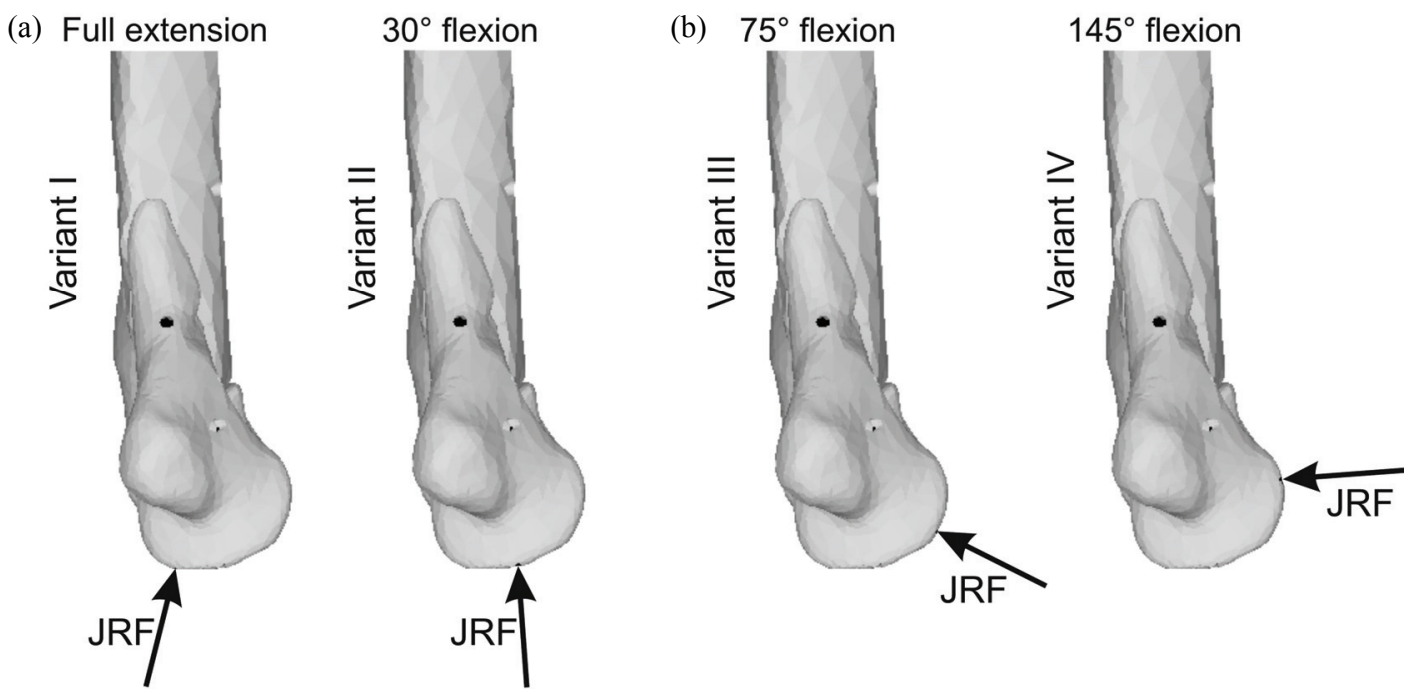

Fig. 4. Joint Reaction Force (JRF) orientation for selected position during elbow flexion (a) with or without lateral or medial deviation in frontal plane (b)

ments (Target170, Contact174). A friction between the bone fragments was taken into consideration (friction coefficient equalled 0.2).

\section{Results}

The values and directions of mutual displacements have been analyzed to assess the conditions of bone fragments stabilizations with regard to different configurations of the stabilizer (Fig. 5a):

a) between the medial and the lateral trochlea fragments (region A),

b) between lengthwise oriented surface of the medial fragment and the shaft (region B),

c) between lengthwise oriented surface of the lateral fragment and the shaft (region C), d) between transversely oriented surface of the lateral fragment and the shaft, in the coronoid fossa region (region $\mathrm{D}$ ).

To simplify the analysis of the dislocations in each of the A-D regions, local, orthogonal coordinate systems were introduced (LCS 1 - LCS 4, Fig. 5b). It was assumed that the $X Y$ surface, covering approximately the gap of the fracture, might be used for the assessment of the tangential displacement between bone fragments (mutual slippage) and the direction of the $Z$ axis reflects normal displacements (approaching each other and moving away from each other).

The maximum values of the normal and tangential mutual displacements between pairs of the bone fragments have been calculated for each of the regions. In the case of normal displacement, the absolute value was taken into account. The overviews of the results obtained for all the three types of stabilization as well (a)

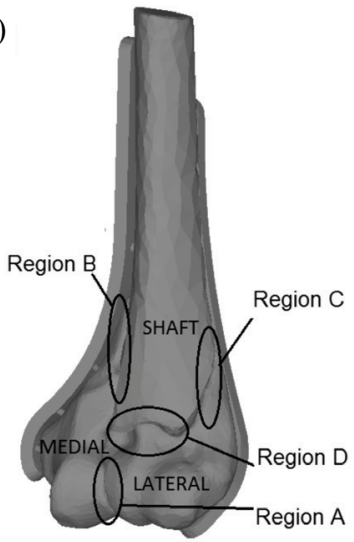

(b)

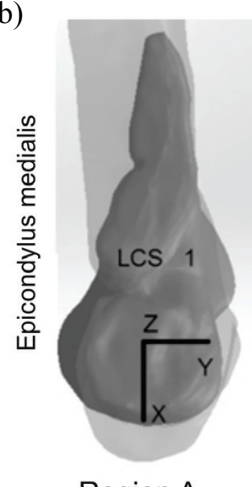

Region A

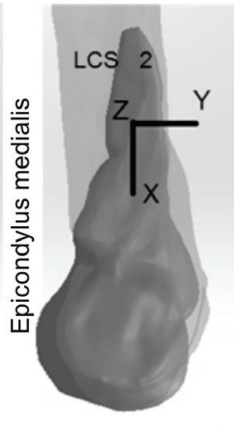

Region B

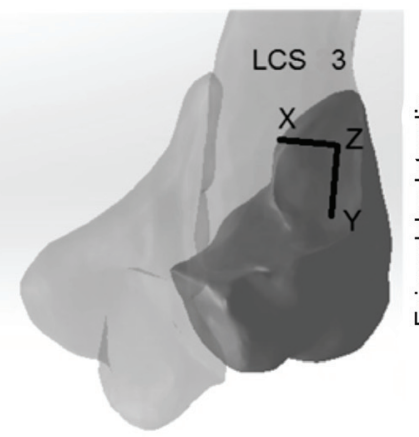

Region C

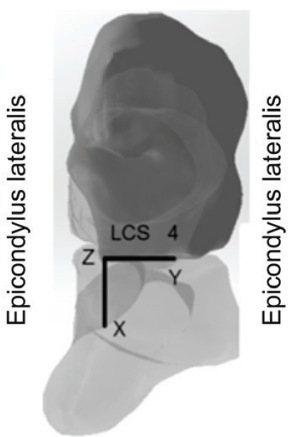

Region D

Fig. 5. The regions A, B, C and D (a) and local coordinate systems (LCS) (b) used for analysis of the mutual displacement of the bone fragments 
as for different angular positions in the elbow joint are presented in Fig. 6.

There are insignificant values of mutual displacements of the bone fragments in the A region (intraarticular zone in the trochlea region) (Fig. 6a). Normal displacements do not show significant changes in the function of the joint angle, with the maximum value of about $0.03 \mathrm{~mm}$. Tangential displacements in this region are slightly higher. Besides, they exhibit more variabil- ity with the flexion of the elbow. The value for the joint angle of $145^{\circ}$ was noted as the highest $(0.07 \mathrm{~mm})$. Taking into account the values of the displacement occurring in this region of fracture and concerning a variety of stabilization variants, no considerable differences were observed.

Displacements between the medial fragment and the shaft (B region, Fig. 6b) are higher than in the trochlea region. Furthermore, they present a slightly

(a) $[\mathrm{mm}] \quad$ REGION A
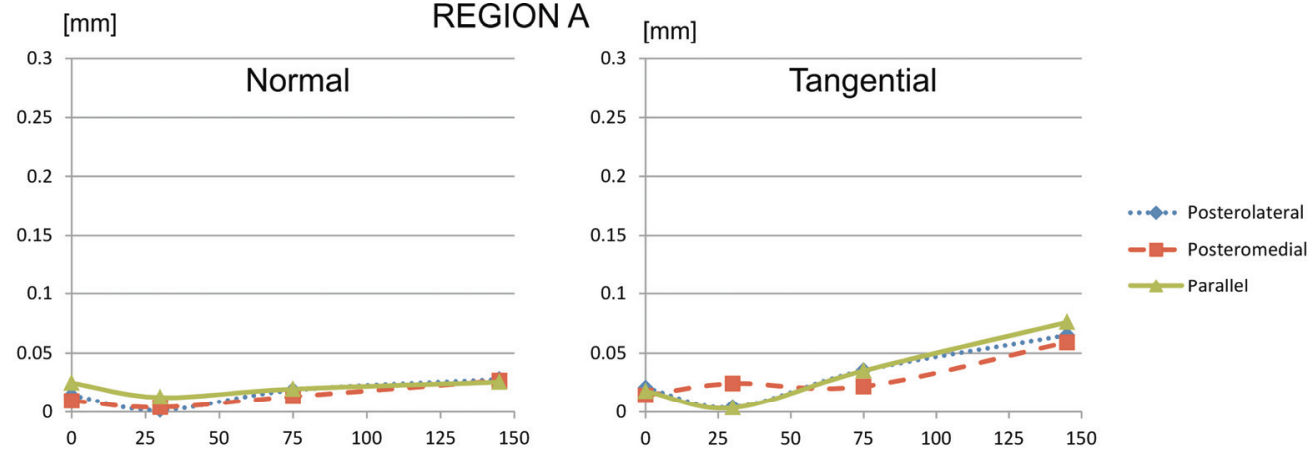

(b)
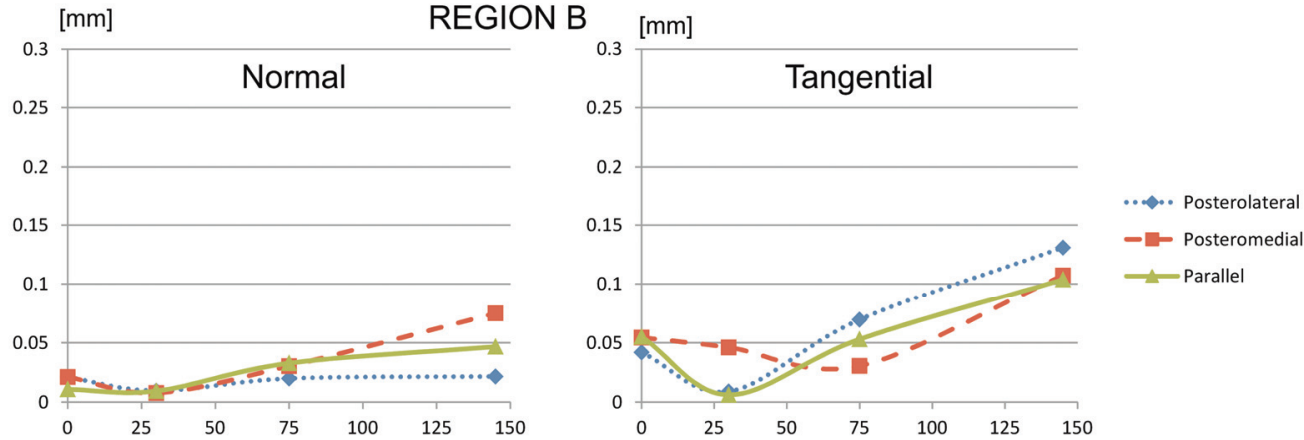

(c)
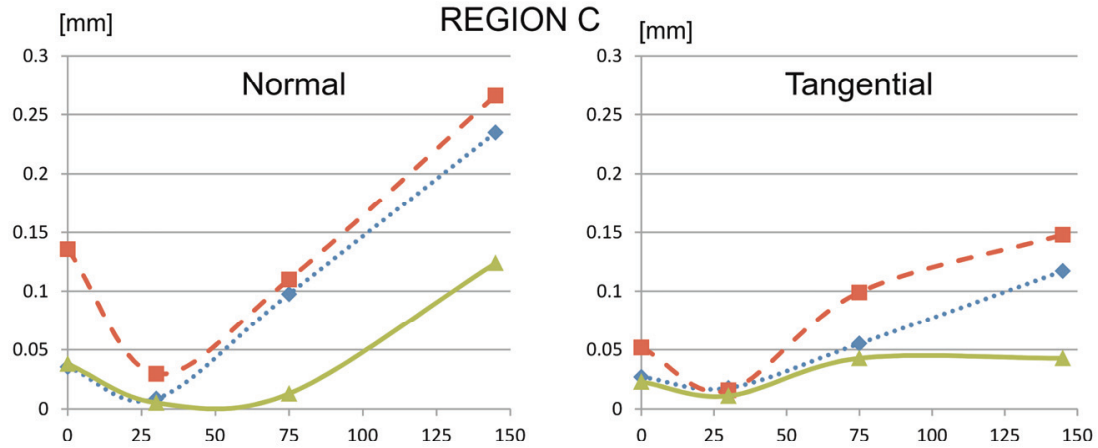

..... Posterolateral

- Posteromedial - Parallel

(d)
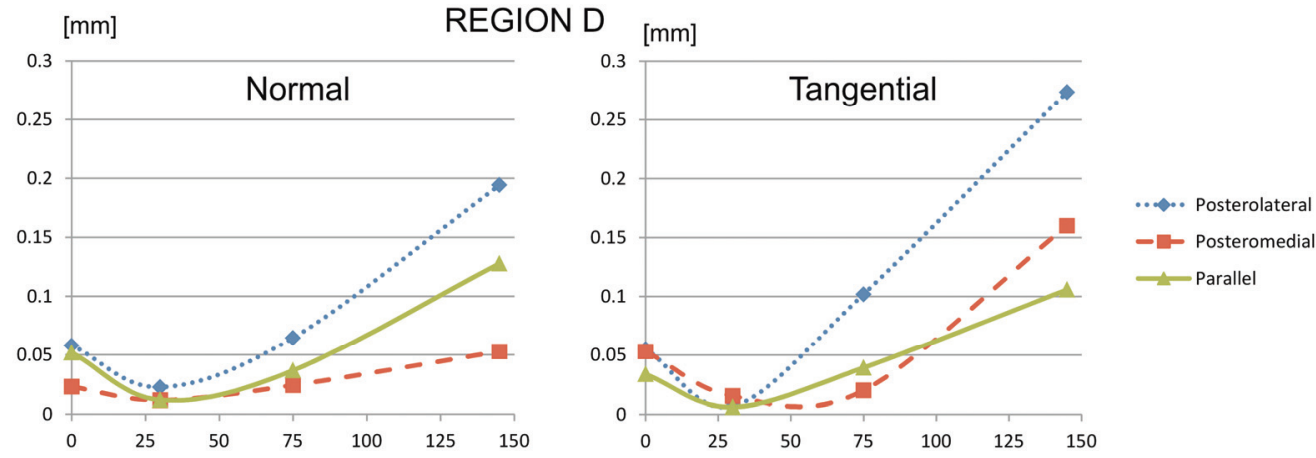

Fig. 6. The maximum mutual displacements of bone fragments in the normal (on the left) and tangential direction (on the right) as regards three methods of stabilization with respect to the flexion angle in the elbow joint: a) region $\mathrm{A} ; \mathrm{b}$ ) region $\mathrm{B} ; \mathrm{c}$ ) region $\mathrm{C} ; \mathrm{d}$ ) region $\mathrm{D}$ 
higher variability with regard to different stabilization methods. The maximum value of tangential displacements, which is $0.13 \mathrm{~mm}$, was noted in the case of posterolateral configuration. As far as this region is concerned, the minimum normal displacements were obtained with this setting of the plates. Additionally, they remained almost unchanged in the whole range of values of the joint angle. For the two remaining configurations, higher values as well as a higher variability of normal displacements were noted (max 0.07 $\mathrm{mm})$. Tangential displacements were significantly changing along with the angle in the elbow joint, reaching their minimum at the angles of $30^{\circ}$ and $75^{\circ}$ (posteromedial), while their maximum was noted at $145^{\circ}$ (the maximum elbow flexion).

The values of normal and tangential displacements, which are substantially higher than the values analyzed so far, take place between the lateral fragment and the shaft (C region - Fig. $6 \mathrm{c}$ ). Normal displacements reach their minimum at the angle of $30^{\circ}$. They are markedly increasing for higher values of the flexion angle in the elbow joint, obtaining the value of about $0.25 \mathrm{~mm}$ for the maximum flexion. Noteworthy is the maintenance of the parallel plate setting for which normal displacements are significantly lower (about three times) in comparison with the both types of perpendicular configurations. In the case of tangential displacements, the advantage of the parallel stabilizer is particularly noticeable for higher values of the joint angle $\left(75-145^{\circ}\right)$.

Considerable values of displacements appear also between the lateral fragment and the shaft at the level of the coronoid fossa (D region, Fig. 6d). The highest values of normal $(0.2 \mathrm{~mm})$ and tangential displacements $(0.27 \mathrm{~mm})$ are observed for the posterolateral configuration regarding the maximum flexion in the elbow joint. As far as smaller values of the joint angle are concerned (between 0 and $30^{\circ}$ ), bone fragments' displacements in this region are minor for all three configurations of the stabilizer and are markedly increasing for higher values of the joint angle. The posteromedial configuration provides the highest stability of bone fragments in the normal direction with regard to this region. Taking tangent displacements into ac-

\section{MEDIAL-LATERAL}

(a) $[\mathrm{mm}]$

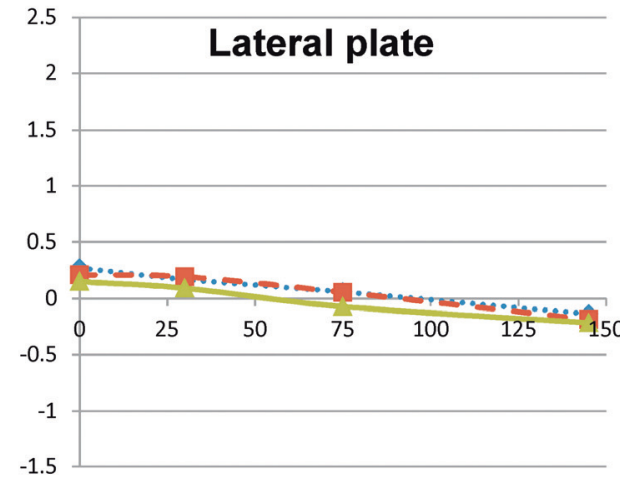

$[\mathrm{mm}]$

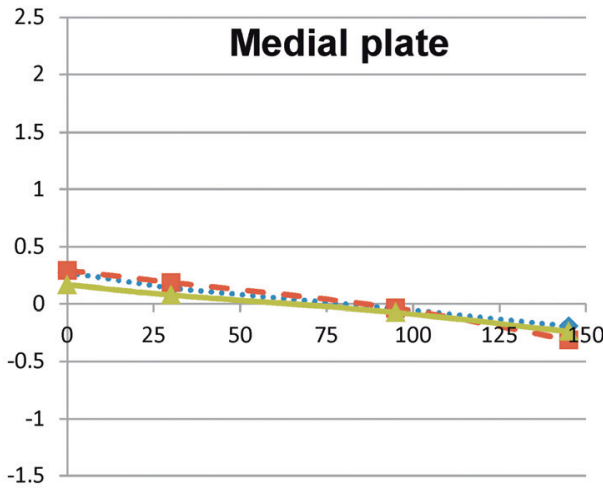

(b)

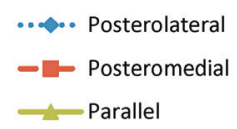

Elbow flexion [ ${ }^{\circ}$ ]

\section{POSTERIOR - ANTERIOR}

(c) $[\mathrm{mm}]$

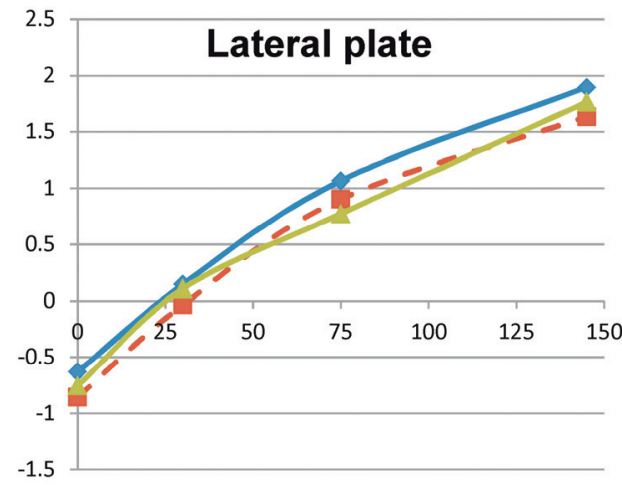

[mm]

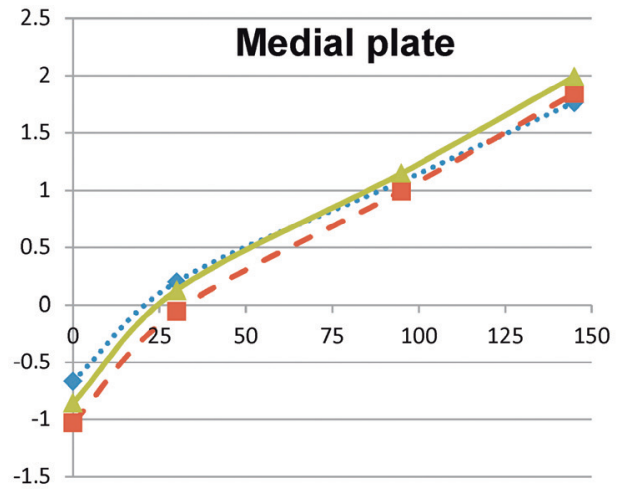

(d)

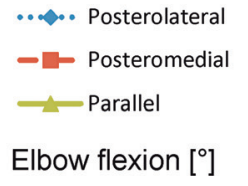

Elbow flexion $\left[{ }^{\circ}\right]$

Fig. 7. The maximum displacements of the lateral $(a, c)$ and medial $(b, d)$ plate, in its distal end, for the three configurations of stabilizer (posterolateral, posteromedial, parallel) as a function of the flexion angle in the elbow joint 
count, this type of plate setting brings satisfactory stabilization results for the joint angle lower than $75^{\circ}$. Nonetheless, the conditions of stabilization are clearly deteriorating when it comes to the maximum flexion.

The deformation of the humerus shaft-plate stabilizer system was analyzed, except for mutual displacements of bone fragments. For this purpose, the maximum displacement of distal end of particular plates was calculated for coronal plane (medial-lateral direction) as well as sagittal plane (posterior-anterior direction). The results are shown in Fig. 7, assuming that, respectively, a positive value stands for the displacement of the distal part of the plate in the medial or posterior direction, and a negative value in the lateral or anterior direction. The results show that the configuration of the stabilizer has no significant impact on the plates' deflection reaching comparable values in all cases. It is also noticeable that the maximum values of the plates' deflection are changing almost linearly as a function of the flexion angle in the elbow joint. A greater deflection of the plates occurs in the sagittal plane (Figs. 7c, d), reaching the value of about $2 \mathrm{~mm}$ for the maximum flexion in the elbow joint $\left(145^{\circ}\right)$.

\section{Discussion}

Previous studies, attempting to evaluate the stability of distal humerus fracture fixation, were based primarily on the estimation of global stiffness of the bone-stabilizer system as well as on the deformity of the plates [8], [14], [22], [26]. Penzkofer et al. [18] and Kudo et al. [14] assessed the change in distances between bone fragments, nevertheless, their analysis took into account only displacements between the shaft and the distal part of the bone without a realistic analysis of the position of particular bone fragments. Bogataj et al. [4] conducted similar research with the use of numerical methods. However, in that case, the fracture was modeled in the form of a rupture between the shaft and the epiphyses without realistic restoration of the interaction between particular bone fragments.

Considering the global stiffness of the bone (stabilizer system as well as the plates' deformation) it is easy to conclude that there are no specific differences between particular configurations of the stabilizer (Fig. 7) and all existing variants show a satisfactory stiffness. The result of the presented numerical analysis, formulated in this manner, is consistent with the findings of previous studies presented by Got et al. [8], Koonce et al. [12] and Schwartz et al. [22]. All above-mentioned studies showed a similar plates' deformation for both parallel and perpendicular configurations. The results of the numerical analysis obtained during the present research clearly demonstrate that the conditions of particular fragment stabilizations vary according to different stabilizer configurations. They may have been noticed only by comparing mutual displacements of bone fragments, being parameters extremely crucial from the point of view of bone fracture healing. Such noticeable differences have their sources in:

- different location and orientation of the bone screws used to stabilize various fragments in relation to the loading direction and the course of the fracture gaps,

- different length of the screws,

- variable conditions of screw' fixation in a bone (support in the other bone fragment or the lack of support, different strength of bone tissue),

It is obvious that the position and the direction of insertion of particular screws into the bone as well as the possibility of interaction with bone fragments depend significantly on the plate position in relation to the bone, the shape of the plate and the location of the screw holes.

The analysis of the results shows that the main difficulty in stabilization and the inconstancy of support conditions occur in the case of the lateral fragment. It is particularly reflected in higher values of displacements as well as in their considerable variability as a function of joint flexion angle and stabilizer configurations (Figs. 6c, d). The lateral fragment clearly moves further away and slides relatively to the bone shaft, especially at the elbow flexion angles above $75^{\circ}$. This creates the risk of a delay or the lack of bone fracture healing. Larger values of displacements of the lateral fragment are caused by insufficient support which results from the plate position, and, consequently, from the localization of the screws inserted in the lateral fragment. Such effect was also observed by Kudo et al [14] as result of biomechanical tests. Using the "posterolateral" configuration where the greatest, normal and tangential displacements are observed, the lateral bone fragment is fixed to the posterolateral plate. Its position and shape required the use of short screws (Fig. 2b) which far ends cannot be anchored in any other bone fragment. As a consequence, the screws fixing the lateral bone fragment anchored unilaterally in a plate and passing only through a quite thin layer of weak cancellous bone (Fig. 3) are not capable of preventing the excessive motion of the bone fragment. A slightly better stabilization of the lateral fragment was obtained in the case of "posteromedial" and 
"parallel" configurations. The lateral plate, which allows for the insertion of longer screws, is used in both cases. The screws are anchored in a plate at one end, while they enter the medial fragment after passing through the lateral fragment at the other end. This leads to interactions between both bone fragments as well as between both plates of the stabilizer. The effects of these interactions are mostly visible in the parallel setting of the stabilizer plates that ensures the best stabilization of the lateral fragment. As far as this configuration is concerned, the relatively long screws that have been inserted through the medial and lateral fragments from each side of both plates (Fig. 2a) mutually block any fragment displacements. This stiffens the entire fixation, creating a closed load-bearing structure resembling the triangle. The setting of the plates together with the bone screws works here as pliers applying pressure on the bone fragments and ensuring a relatively high stability of the structure. Such observations are consistent with the conclusions of O'Driscoll [17] and Jung et al. [10] who noticed that the closed arch being a mechanism ensuring an equitable support and stabilization of the system is formed when connecting the lateral column with the medial column by means of the screws.

Based on the findings of the numerical analysis, it may be concluded that better conditions of stabilization were obtained for the medial fragment when it was stabilized with the use of a larger number of screws, and, in addition, some of them were constrained in both the medial plate and the humeral bone shaft (Fig. 2). Such location of the screws is more favorable, as the support of the screw in the shaft of humerus reduces the length of the force arm trying to destabilize the medial fragment. It seems that this observation is in accordance with the thesis of O'Driscoll [17] that the fixation of distal fragments of humerus should be based on the connection of bone fragments with the plate, and at the same time, with the bone shaft. An important argument in favor of such thesis is that, according to the CT data and Diederichs's [7] observations, the bone tissue is stronger in the area of the medial fragment. Thus, the screws are more strongly anchored and it is possible to obtain a better fragment stabilization. The influence of the bone quality on durability of the screw fixation was shown also by MacLeod et al. [15].

All plate configurations ensure a proper stabilization in the articular region, which is the lateral fragment relative to the medial fragment in the trochlea region (Fig. 6a). It is very important due to the fact that eliminating the mutual displacements of the fragments comprising the joint surface is decisive in the case of intra-articular fractures to ensure conditions for direct healing without external callus. A substantial number of long screws passing from one bone fragment to another through the fracture gap helps minimize the mutual displacements for all stabilizer types.

Although there are some differences noticeable in particular stabilizer configurations and regions of observation, what must be emphasized is the crucial impact of direction of the force acting on the distal humerus affecting mutual displacements of the fragments. The most advantageous conditions of stabilization occur when the force acts more or less along the shaft of the humerus which, in line with the used model, reflects the flexion of $30^{\circ}$ in the elbow joint (Fig. 4). Both the full extent and the greater flexion in the joint cause an increase of mutual displacements of the bone fragments (Fig. 6). The obtained findings are consistent with the reports of Schwartz et al. [22] and Penzkofer et al. [18] who observed that the greatest stiffness of the stabilizer occurs in the case of axial loadings while a significant decrease (7-10 times) of structure stiffness is noticeable in the case of loads acting perpendicularly to the humerus axis. The stabilizer with the parallel plates is most resistant to the change in loading direction. This can be explained by the fact that the parallel setting of both plates towards each other as well as towards the plane comprising the joint force direction makes these configurations more resistant to flexion.

When assessing the achieved results of numerical simulation, it should be remembered that the analysis was performed for constant values of the joint loads. However, based on the analysis of Kincaid et al. [11], the reaction in the elbow joint together with the change of the elbow flexion angle change not only its direction, but also its value, reaching the maximum for the full extension. Such effect has not been taken into consideration in the present study, which made it possible to conduct a comparative analysis of a variety of stabilizer configurations under the influence of the loads of various directions.

It must be also remembered that numerical model should be validated. For that reason, further experiment with use of Sawbones artificial bone models and their numerical equivalents is planned.

\section{Conclusions}

The findings obtained during the research have shown that: 
- conditions of particular bone fragments' stabilization depend not only on the stiffness of the plates used, but also on the manner in which the fragments are supported with the screws. The noticeable variability of bone fragments displacements, in the light of sufficient stiffness of all the stabilizer configurations, result from different possibilities to immobilize the bone fragments with the use of different spatial arrangement and shape of the plates;

- due to a multi-directional position of several screws in all stabilizer configurations, there are favorable conditions to obtain a stable fixation of the articular fragments;

- the most difficult conditions of stabilization occur in the case of the lateral bone fragment;

- the parallel configuration provides a better stability of various bone fragments for most embodiments of the load, but does not guarantee the optimum conditions of union of all fragments for each of the elbow flexion positions.

The conducted study has clearly proved that classical tests assessing the global stiffness of the stabilizer are insufficient to evaluate the stabilization conditions of comminuted fractures of distal humerus. More effective research of stabilization conditions is only possible when the mutual displacements between bone fragments considering the spatial configuration of the bone-stabilizer-loadings system will be analyzed. Modeling and numerical simulation seem to be the best methods of seeking optimal stabilization methods for particular clinical cases. Nevertheless, the aim should be to consider a three-dimensional assessment of particular bone fragments' displacements also in experimental studies.

\section{Acknowledgements}

The research was performed as a part of the project $\mathrm{S} / \mathrm{WM} / 1 / 2017$ and was financed with the funds for science from the Polish Ministry of Science and Higher Education.

\section{References}

[1] Amir S., Jannis S., DANiEl R., Distal humerus fractures: a review of current therapy concepts, Curr. Rev. Musculoskelet. Med., 2016, 9, 199-206.

[2] Arnander M.W., Reeves A., MacLeod I.A. et al., A biomechanical comparison of plate configuration in distal humerus fractures, J. Orthop. Trauma, 2008, 22, 332-336.

[3] Black J., Hastings G., Titatium and titanium alloys, [in:] J. Black, G. Hastings (Eds.), Handbook of biomaterial properties, Chapman \& Hall, 1998, 179-200.
[4] Bogataj M., Kosel F., Norris R. et al., Biomechanical study of different plate configurations for distal humerus osteosynthesis, Med. Biol. Eng. Comput., 2015, 53, 381-392.

[5] CALIŞAL E., UĞUR L., Evaluation of the plate location used in clavicle fractures during shoulder abduction and flexion movements: a finite element analysis, Acta Bioeng. Biomech., 2018, 20 (4), 41-46.

[6] Clavert P., Ducrot G., Sirveaux F. et al., Outcomes of distal humerus fractures in patients above 65 years of age treated by plate fixation, Orthop. Traumatol. Surg. Res., 2013, 99, 771-777.

[7] Diederichs G., ISSEVer A.S., Greiner S. et al., Three-dimensional distribution of trabecular bone density and cortical thickness in the distal humerus, J. Shoulder Elb. Surg., 2009, 18, 399-407.

[8] Got C., Shuck J., Biercevicz A. et al., Biomechanical comparision of parallel versus 90-90 plating of bicolumn distal humerus fractures with intra-articular comminution, J. Hand Surg., 2012, 37A, 2512-2518.

[9] GREIWE R.M., Distal humerus fractures: open reduction and internal fixation, [in:] M. Greiwe (1st Ed.), Shoulder and Elbow Trauma and its Complications, Vol. 2: The Elbow, Woodhead Publishing, 2016, 65-97.

[10] Jung S.W., Kang S.H., JeOng M., Lim H.S., Triangular fixation technique for bicolumn restoration in treatment of distal humerus intercondylar fracture, Clin. Orthop. Surg., 2016, 8, 9-18.

[11] KINCAID B.L., AN K.-N., Elbow joint biomechanics for preclinical evaluation of total elbow prostheses, J. Biomech., 2013, 46, 2331-2341.

[12] KoOnCe R.C., Baldini T.H., Morgan S.J., Are conventional reconstruction plates equivalent to precontoured locking plates for distal humerus fracture fixation? A biomechanics cadaver study, Clin. Biomech., 2012, 27, 697-701.

[13] Kruszewski A., Piszczatowski S., Piekarczyk P., KWIATKOWSKI K., Biomechanical evaluation of the stabilization used in the treatment of the distal humerus intra articular fractures, IFMBE Proc., 2015, 50, 75-78.

[14] Kudo T., Hara A., Iwase H., Ichihara S., Nagao M., MARUYMA Y. et al., Biomechanical properties of orthogonal plate configuration versus parallel plate configuration using the same locking plate system for intra-articular distal humeral fractures under radial or ulnar column axial load, Injury, 2016, 47, 2071-2076.

[15] Macleod A.R., Simpson A.H., Pankaj P., Age-Related Optimization of Screw Placement for Reduced Loosening Risk in Locked Plating, J. Orthop. Res., 2016, 34, 18561864.

[16] MurRay I.A., Johnson G.R., A study of the external forces and moments at the shoulder and elbow while performing everyday tasks, Clin. Biomech., 2004, 19, 586-594.

[17] O'DrisColl S.W., Optimizing stability in distal humeral fracture fixation, J. Shoulder Elb. Surg., 2005, 14 (1, Suppl. S), 186-194.

[18] Penzkofer R., Hungerer S., Wipf F. et al., Anatomical plate configuration affects mechanical performance in distal humerus fracture, Clin. Biomech., 2010, 25, 972-978.

[19] Puchwein P., Wildburger R., Archan S. et al., Outcome of type $C(A O)$ distal humeral fractures: follow-up of 22 patients with bicolumnar plating osteosynthesis, J. Shoulder Elbow Surg., 2011, 20, 631-636.

[20] Rho J.Y., Hobatho M.C., Ashman R.B., Relations of mechanical properties to density and CT numbers in human bone, Med. Eng. Phys., 1995, 17, 347-355. 
[21] Ruedi T.P., Buckley R., Moran C.G., AO principle of Fracture management, Thieme, Stuttgart 2007.

[22] Schwartz A., OKa R., Odell T. et al., Biomechanical comparison of two different periarticular plating systems for stabilization of complex distal humerus fracture, Clin. Biomech., 2006, 21, 950-5.

[23] SHIN S.J., SoHn H.S., Do N.H., A clinical comparison of two different double plating methods for intraarticular distal humerus fracture, J. Shoulder Elb. Surg., 2010, 19, 2-9.

[24] Schmidt-Horlohe K.H., BonK A., Wilde P. et al. Promising results after a treatment of simple and complex distal humerus type $C$ fractures by angular-stable double-plate osteosynthesis, J. Orthop. Trauma, 2013, 99, 531-541.

[25] Theivendran K., Duggan P.J., Deshmukh S.C., Surgical treatment of complex distal humeral fractures: Functional outcome after internal fixation using precontoured anatomic plates, J. Shoulder Elbow Surg., 2010, 19, 524532.

[26] Zalavras C.G., Vercillo M.T., Bong Jae J. et al., Biomechanical evaluation of parallel versus orthogonal plate fixation of intra-articular distal humerus fractures, J. Shoulder Elb. Surg., 2011, 20, 12-20. 